LAWS OF ATMOSPHERIC MOVEMENTS. ${ }^{1}$ THE motion of the upper layers of the atmosphere is discussed in these two papers by Dr. W. N. Shaw, recently published. It is difficult in a short space to give a clear idea of the conclusions reached, but some of the main points may be here summarised.

In the paper published by the Scottish Meteorological Society it is shown that if $p$ denote the pressure in millibars, and $\theta$ the absolute temperature (C.), and $\Delta p$ and $\Delta \theta$ the changes that occur in passing horizontally from one place to another, then the rate of increase of pressure difference in millibars per metre of height is $0.0342 p / \theta(\Delta \theta / \theta-\Delta p / p)$. It is then shown that from about $I \mathrm{~km}$. to $9 \mathrm{~km}$. the values of $\Delta \theta$ and $\Delta p$ have in general the same sign, so that the term in brackets is small, and hence pressure differences, i.e. the barometric gradient, are maintained without much alteration up to $9 \mathrm{~km}$. Above Io or II km. $\Delta \theta$ and $\Delta p$ have in general a different sign and the magnitude of the gradient rapidly falls off. The effect upon the wind at various altitudes is then considered, and special cases where simultaneous observations over England, Scotland, and Ireland are available are taken.

In the paper published by the Royal Society of Edinburgh, Dr. Shaw gives five axioms or laws of atmospheric motion, two lemmas or postulates, and six propositions. His first law reads thus:-

"In the upper layers of the atmosphere the steady horizontal motion of the air at any level is along the horizontal section of the isobaric surfaces at that level, and the velocity is inversely proportional to the separation of the isobaric lines in the level of the section."

The whole discussion turns upon the truth of this law, and Dr. Shaw confesses that observation is at present incapable of proving or of disproving it. There can be very little doubt that it is approximately true, for except near the equator pressure differences come into existence and persist for days or even weeks. These differences could not continue even for an hour if there were not some compensating horizontal acceleration acting on the air from the low towards the high pressure, for otherwise the inevitable rule which makes the surface of a liquid horizontal would come into play, and a depression would be filled up almost as soon as it was formed. Some opposing acceleration must therefore act whenever and wherever there is a barometric gradient, and we can conceive of no other possible source of this acceleration save that given in law I. But in the upper strata there must be a certain small amount of flow outward across the isobars from low to high pressure to compensate for the inverse flow that occurs close to the earth, where frictional resistances prevent the requisite velocity along the isobars from being attained. The other laws and the two lemmas will probably be accepted with the small reservations given by the author without demur.

The propositions follow from the laws and postulates. They are of great interest, but are too long to be quoted here. It will suffice to say that Dr. Shaw finds that a current flowing east or west will be stable, but a current flowing north or south is more or less unstable, and must lose or gain air as it goes. Also his suggestion about the flow of air up or down the land slopes from the interior of the continents to the sea is very pertinent, and, to my mind, affords a better explanation of the winter anticyclone over Asia and North America than that commonly given.

$$
\text { W. H. Dines. }
$$

I (x) "Upper Air Calculus and the British Soundings during the International Week (May 5-10), rar3." From the Journal of the Scottish Meteorological Socrety. Third series. Vol. xvi. No xxx.

(2) "Principia Atmospherica: a Study of the Circulation of the Atmosphere." (Proc. Roy. Soc. Edin., vol. xxxiv., 19r4, pp. 77-rir).

NO. 2324, VOL. 93]

\section{AN ELECTRICAL ANALOGY OF THE} ZEEMAN EFFECT.

THE discovery, announced by J. Stark in Nature 1 of December 4 , 1913, that when hydrogen in a state of luminescence is placed in an electric field of suitable strength and direction, the spectral lines are resolved into three or more components, is evidently a fact of prime physical importance. It will place in the hands of physicists another method of investigating the internal structure of the atom, and, in conjunction with the Zeeman effect, will no doubt be of immense service in the discovery of further regularities in spectral series, and in the attempts now being made by Bohr, Nicholson, and others to explain the origin of spectra on a dynamical basis. In this connection a series of papers in the Rendiconti della R. Accad. dei Lincei by Garbasso, Lo Surdo, and Puccianti will be of great interest to readers of NATURE.

The effect appears to have been observed independently by Lo Surdo whilst working on the retrograde positive rays in the neighbourhood of the kathode. An account of his first observations is given in a paper read on December 21, 1913. A cylindrical tube $20 \mathrm{~cm}$. long and $4 \mathrm{~mm}$. in diameter was used. It carried disc electrodes which completely filled the section, and it was excited by means of an accumulator battery. In these circumstances it was found that the electric field in the Crookes dark space was of itself sufficient to produce resolution of the lines. The observations were made with a four. prism quartz spectrograph. By suitable modifications the tube was varied so that the line of sighit was either along or perpendicular to the field. The two outer components are polarised with the electric vector parallel to the field, the remainder in a perpendicular plane. When observations are made along the lines of force the outer components are missing. In a later paper, using a tube $\mathrm{r} .5 \mathrm{~mm}$. in diameter, Puccianti finds that the effect can readily be seen in the well-known Hilger wave-length spectroscope with constant deviation prism. The lines of the hydrogen spectrum show an interesting series of regularities; these are displayed in the following tabie (after Lo Surdo). Writing the Balmer formula in the form, $\mathrm{r} / \lambda=a-4 a / n^{2}, n$ has the values 3,4 , etc., for the different lines.

Total no. of components

Order of line in the series $\ldots \begin{array}{llll}\ldots & \ldots & \ldots\end{array}$ Component with electric
vector $\perp$ to the field...

Appearance of resolved) $\begin{array}{lllll}\text { lines }^{1} & \ldots & \ldots & \ldots & \ldots\end{array}$

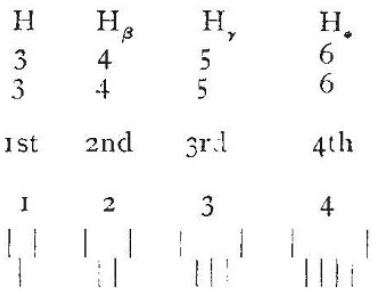

It is seen that the number of components in a given line is the same as the corresponding value of $n$ in the Balmer formula, and that the number of internal components is the same as the order of the given line in the spectral series. According to the measurements of Puccianti the separation of the outer components for $\mathrm{H}_{\beta}$ and $\mathrm{H}_{a}$ are in the ratio $\mathbf{x} \cdot 49$, or, expressed as fractions of the corresponding wave-lengths,

$$
\frac{\delta_{\beta}}{\lambda_{\beta}} / \delta_{a}=2 \cdot 0 \mathrm{I}
$$

In a paper of December $2 x$, 1913, Garbasso dis cusses the matter theoretically, to arrive at the conclusion that the Thomson model atom is incapable of explaining the (earlier) observations, except by the introduction of improbable hypotheses.

R. S W,

1 The components placed above have the vector parallel to the field. 